\title{
Local Graft Irradiation for Kidney Allograft Rejection: A Case Series and Review of the Literature
}

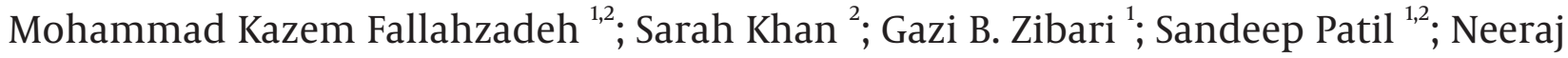 \\ Singh ${ }^{1,2, *}$ \\ John C. McDonald Regional Transplant Center, Willis Knighton Health System, Shreveport, USA \\ 2 Division of Nephrology, Department of Medicine, LSUHSC-S, Shreveport, USA \\ ${ }^{*}$ Corresponding author: Neeraj Singh, Division of Nephrology, Department of Medicine, LSUHSC-S, 1501 Kings Highway, Shreveport, USA. Tel: +1-3182128386, Fax: +1-3182124957, E-mail: \\ nsing1@lsuhsc.edu
}

Received: November 19, 2013; Accepted: November 30, 2013

\begin{abstract}
Introduction: Due to its immunosuppressive properties, local graft irradiation (LGI) has been proposed as a second line therapy for treatment of acute kidney rejection.

Case Presentation: In this case-series we report 6 patients with biopsy proven acute kidney allograft rejection refractory to conventional antirejection therapy who underwent LGI for treatment of acute rejection at our center. Three of these patients had living donor transplants, 2 had deceased donor transplants, and one had received a simultaneous kidney/pancreas transplant. All patients were treated with anti thymocyte-globulin or muromonab-CD3, and intravenous steroids for initial treatment of rejection. Three patients also received intravenous immunoglobulin. LGI was tried as a last resort and was well tolerated and resulted in either improvement or stabilization of renal function in 5 patients. One patient could not be given the complete course of chemical immunosuppression for treatment of rejection due to concomitant cryptococcal meningitis and was switched to LGI with good short-term response.

Discussion: Our results suggest that LGI could be considered a second line therapy to the conventional anti-rejection therapy for patients with refractory acute kidney allograft rejection, or for patients who cannot receive systemic immunosuppression due to severe infection.

Keywords:Rejection, Graft; Immunosuppression; Kidney; Radiotherapy; Transplantation
\end{abstract}

\section{Introduction}

Over the last 40 years, kidney transplantation has become the preferred method of treatment for end-stage renal disease (ESRD). Acute rejection is the most important cause of kidney allograft failure. The conventional anti-rejection therapy includes intravenous steroids and antithymocyte globulin (ATG) $(1,2)$. In cases of antibody mediated rejection, therapies like plasmapheresis, intravenous imunoglobulin (IVIG), rituximab and/ or bortezomib are often added (1-3). Local graft irradiation (LGI) is a lesser known therapy for the treatment of rejection. The immunosuppressive properties of the radiation therapy have been known for a long time. These properties are currently harnessed to eliminate lymphocytes in blood transfusion products in order to prevent transfusion associated complications like graft versus host disease (4). Radiation therapy has also been used in the field of renal transplantation since its early days (5). Initial studies showed improved graft survival with different methods of radiation such as extracorporeal blood irradiation (6), total lymphoid irradiation (7) and local graft irradiation (LGI) (8). Despite the initial promising results in small retrospective studies (8), later randomized trials did not show the beneficial effects of LGI for treatment of acute allograft rejection as first line adjunct therapy added to a conventional antirejection regimen like antithymocyte globulin (ATG) and steroids (9-11). However, the efficacy of LGI as a second line therapy after failure of conventional antirejection treatment has been more successful (12-17). In this study, we have reported our experience with 6 patients with acute kidney allograft rejection who were treated with LGI as a second line therapy. In addition, we have reviewed the previous literature on the use of LGI for acute kidney allograft rejection. This study was approved by Institutional Review Board of our institution and was done in accordance with the Helsinki Declaration of 1975 (as revised in 1983). In this study the charts of all patients with acute kidney allograft rejection who were treated with LGI at our center were retrospectively reviewed. Rejection refractory to medical therapy was defined as an acute rejection that did not respond to conventional anti-rejection therapy (antithymocyte 
globulin [ATG] or muromonab-CD3 per physician's discretion in addition to intravenous steroids) with failure of serum creatinine to trend down until at least 1 week after the completion of anti-rejection therapy. IVIG was also given to patients with positive donor specific antibody (DSA) levels which were defined as a mean fluorescence intensity $\geq 2000$. The DSA level were measured by a Luminex analyzer (Luminex Corporation, Austin, TX, USA). Episodes of acute rejection were confirmed by renal biopsy. The dose range of LGI treatment varied from 600-800 cGy given in the fractions of 150 cGy to 200 cGy daily for 4 days using either 6MV and/or 18MV photons.

\section{Case Presentation}

The patients' demographics, clinical characteristics, radiotherapy doses and final outcomes are shown in Table 1. Per our center's protocol all patients except patient No 4 received 7-10 doses of ATG (1.5 mg/kg IV daily) or 10 doses of muromonab-CD3 (5 mg IV daily) along with high dose IV steroids for treatment of acute allograft rejection. Except for patient No 6, the kidney function improved temporarily in all patients who were treated with LGI. Our patients' kidney allograft survival at 1, 3, 6 and 12 months was 83\%, 83\%, 33\% and $17 \%$, respectively. Patient No 4 was diagnosed with cryptococcal meningitis 3 days after biopsy proven allograft rejection, and therefore he was treated with IV steroids and ATG for only 3 days. He was switched to LGI on day 5 with good response.

\section{Discussion}

Our results suggest that for patients with refractory acute kidney allograft rejection LGI may be considered as a second line therapy to conventional antirejection therapy including intravenous steroids and ATG. In our study, 5 out of 6 patients with refractory rejection responded favorably to LGI. In addition, patient No.4 had concomitant acute rejection and cryptococcal meningitis and responded well to LGI suggesting that LGI may also be indicated for patients with acute rejection when systemic immunosuppression is contraindicated as in the setting of superimposed serious systemic infection. In our study, LGI was found to be safe and without any major adverse effect. Although the initial reports on using LGI as an adjuvant therapy for acute rejection were encouraging (8), 3 later randomized trials did not show the efficacy of LGI as an addition to the chemical immunosuppressive therapies in the first line of treatment for acute kidney rejection (9-11). Later studies showed that LGI could be used as a second line therapy when the acute kidney rejection failed to respond to medical immunosuppressive therapy (Table 2). In 1984, Hal- perin et al. (12) demonstrated that in 53 patients with acute allograft rejection resistant to medical therapy, radiotherapy resulted in improvement or stabilization of renal function in $42 \%$ of the patients a month after LGI. But one year post-LGI, only $21 \%$ of these allografts were still functional. In this study, 10 patients with acute allograft rejection in whom immunosuppressive therapy was contraindicated because of either systemic infections or hematologic dyscrasias received LGI; 1 month and 1 year graft survival rates in this group were $90 \%$ and $40 \%$, respectively. Later, Noyes et al. (14) and Nuyttens et al. (15) reported a 1 year graft survival of $49 \%$ and $50 \%$, respectively, in patients who had acute rejection refractory to medical therapy and who received LGI. In a study by Chen et al. (17) which mostly included patients with acute allograft rejection resistant to medical therapy, 1 year graft survival rate was $60 \%$. Wahl et al. (16) reported a 1 month graft survival of $63 \%$, but the 1 year graft survival was poor at $31 \%$. Similar to previous reports, our study also shows a good short term graft survival (83\% at 1 and 3 months) but poor long term graft survival (17\% at 1 year) when LGI is given as a second line agent for refractory rejection.

The mechanism of action of LGI in the treatment of rejection appears to be the elimination of lymphocytes. Dividing cells like lymphocytes, both in circulation and in tissues, have been shown to be particularly sensitive to radiation therapy (18). Radiation can directly damage DNA; however, generation of free reactive oxygen species from the radiolysis of water by radiation is the most important cause of DNA damage and cell death. The T-cells involved in acute cellular rejection are located in the transplanted kidney, but plasma cells that produce antibodies involved in antibody mediated rejection are mostly located in the lymph nodes and bone marrow (19). Therefore, by preferentially targeting T-cells located in the kidney, LGI is probably more effective against acute cellular rejection compared with antibody mediated rejection. Compared with sensitive dividing cells like lymphocytes, non-dividing cells like renal cells are less sensitive to radiation therapy (18). Moreover, the dose of radiation in LGI is low. Therefore, LGI appears to be safe in the treatment of acute kidney allograft rejection without major side effects like radiation nephritis $(10,16)$.

LGI may be tried as a second line therapy for treatment of kidney allograft rejection refractory to conventional anti-rejection medical therapy. In addition, LGI may be a useful modality for treatment of kidney allograft rejection when systemic immunosuppression is contraindicated due to concomitant severe and serious infection. The benefits of LGI should be confirmed in future prospective randomized controlled trials. 
Fallahzadeh MKet al.

Table 1. The Demographics, Clinical Characteristics, Radiotherapy Doses and Final Outcomes of Patients With Acute Kidney Allograft Rejection Refractory to Medical Therapy Treated With LGI ${ }^{\text {a }}$

\begin{tabular}{|c|c|c|c|c|c|c|}
\hline Patients No. & 1 & 2 & 3 & 4 & 5 & 6 \\
\hline $\begin{array}{l}\text { Age at time of } \\
\text { transplanta- } \\
\text { tion, } y\end{array}$ & 28 & 26 & 21 & 38 & 32 & 35 \\
\hline $\begin{array}{l}\text { Type of kidney } \\
\text { transplantation }\end{array}$ & Deceased donor & Living donor & Living donor & $\begin{array}{l}\text { Deceased donor } \\
\text { (simultaneous } \\
\text { kidney/pancreas } \\
\text { transplant) }\end{array}$ & Living donor & Deceased donor \\
\hline $\begin{array}{l}\text { Etiology of kid- } \\
\text { ney disease }\end{array}$ & $\begin{array}{l}\text { Hypertensive } \\
\text { nephropathy }\end{array}$ & IgA nephropathy & $\begin{array}{l}\text { Reflux nephropa- } \\
\text { thy }\end{array}$ & $\begin{array}{l}\text { Diabetic ne- } \\
\text { phropathy }\end{array}$ & $\begin{array}{l}\text { Diabetic ne- } \\
\text { phropathy }\end{array}$ & $\begin{array}{l}\text { Hypertensive } \\
\text { nephropathy }\end{array}$ \\
\hline $\begin{array}{l}\text { Baseline serum } \\
\text { creatinine } \\
\text { range, } \mathbf{m g} / \mathbf{d L}\end{array}$ & $2.0-2.4$ & $1.5-2.1$ & $2.5-3$ & $2.5-2.7$ & $1.5-2.1$ & $2.5-3.2$ \\
\hline $\begin{array}{l}\text { Time from } \\
\text { kidney trans- } \\
\text { plantation to } \\
\text { rejection, mo }\end{array}$ & 54 & 22 & 36 & 29 & 75 & 21 \\
\hline $\begin{array}{l}\text { Allograft kidney } \\
\text { biopsy }\end{array}$ & $\begin{array}{l}\text { Grade } 1 \text { A rejec- } \\
\text { tion, Peritubular } \\
\text { capillaritis, C4d+, } \\
\text { No fibrosis }\end{array}$ & $\begin{array}{l}\text { Grade } 1 \mathrm{~B} \text { rejec- } \\
\text { tion, Peritubular } \\
\text { capillaritis, C4d+, } \\
\text { No fibrosis }\end{array}$ & $\begin{array}{l}\text { Grade } 1 \mathrm{~B} \text { rejec- } \\
\text { tion, Thrombotic } \\
\text { microangiopathy, } \\
\text { C4d+, Minimal } \\
\text { fibrosis }\end{array}$ & $\begin{array}{l}\text { Grade } 1 \mathrm{~B} \text { rejec- } \\
\text { tion, } \mathrm{C} 4 \mathrm{~d}-\text {-, Moder- } \\
\text { ate fibrosis }\end{array}$ & $\begin{array}{l}\text { Grade } 1 \text { A rejec- } \\
\text { tion, } C 4 d-\text {, Moder- } \\
\text { ate fibrosis }\end{array}$ & $\begin{array}{l}\text { Grade } 1 \mathrm{~B} \text { rejec- } \\
\text { tion, Peritubular } \\
\text { capillaritis, C4d-, } \\
\text { No fibrosis }\end{array}$ \\
\hline DSA & + & - & + & - & - & + \\
\hline $\begin{array}{l}\text { Medical Manage- } \\
\text { ment }\end{array}$ & $\begin{array}{l}\text { ATG }(1.5 \mathrm{mg} / \mathrm{kg} \\
\times 8 \text { doses }), \mathrm{IVIG} \\
\text { (50 } \mathrm{g} \times 1 \text { dose }), \mathrm{IV} \\
\text { steroids taper }\end{array}$ & $\begin{array}{l}\text { Muromonab-CD3 } \\
\text { ( } 5 \text { mg × } 10 \text { doses), } \\
\text { IV steroids taper }\end{array}$ & $\begin{array}{l}\text { ATG }(1.5 \mathrm{mg} / \mathrm{kg} \\
\times 10 \text { doses }), \text { IVIG } \\
\text { (40 } \mathrm{g} \times 1 \text { dose }), \text { IV } \\
\text { steroids taper }\end{array}$ & $\begin{array}{c}\text { ATG }(1.5 \mathrm{mg} / \mathrm{kg} \times 3 \\
\text { doses }), \text { IV steroids } \\
\text { taper }\end{array}$ & $\begin{array}{c}\text { ATG }(1.5 \mathrm{mg} / \mathrm{kg} \times 7 \\
\text { doses }), \text { IV steroids } \\
\text { taper }\end{array}$ & $\begin{array}{l}\text { ATG }(1.5 \mathrm{mg} / \mathrm{kg} \times \\
10 \text { doses), IVIG (40 } \\
\mathrm{g} \times 2 \text { doses), IV } \\
\text { steroids taper }\end{array}$ \\
\hline $\begin{array}{l}\text { Maintenance } \\
\text { therapy before } \\
\text { rejection }\end{array}$ & $\begin{array}{l}\text { Mycophenolic } \\
\text { acid } 720 \mathrm{mg} \text { BID, } \\
\text { tacrolimus } 16 \mathrm{mg} \\
\text { BID, prednisone } \\
2.5 \mathrm{mg} \text { QD }\end{array}$ & $\begin{array}{l}\text { Mycophenolate } \\
\text { mofetil } 1000 \text { mg } \\
\text { BID, tacrolimus } 2 \\
\text { mg BID, predni- } \\
\text { sone } 2.5 \text { mg QD }\end{array}$ & $\begin{array}{l}\text { Mycophenolate } \\
\text { mofetil } 1000 \text { mg } \\
\text { BID, tacrolimus } 2 \\
\text { mg BID, predni- } \\
\text { sone } 20 \text { mg QD }\end{array}$ & $\begin{array}{l}\text { Sirolimus } 1 \mathrm{mg} \\
\text { QD, tacrolimus } 1 \\
\text { mg BID, predni- } \\
\text { sone } 20 \text { mg QD }\end{array}$ & $\begin{array}{l}\text { Mycophenolate } \\
\text { mofetil } 1000 \text { mg } \\
\text { BID, tacrolimus } 2 \\
\text { mg BID, predni- } \\
\text { sone } 10 \text { mg QD }\end{array}$ & $\begin{array}{l}\text { Mycophenolate } \\
\text { mofetil } 750 \text { mg } \\
\text { BID, tacrolimus } 3 \\
\text { mg BID, predni- } \\
\text { sone } 2.5 \text { mg QD }\end{array}$ \\
\hline Prior Rejections & 4 & 3 & 3 & 6 & 4 & 0 \\
\hline $\begin{array}{l}\text { Time difference } \\
\text { between biopsy } \\
\text { and radiother- } \\
\text { apy, d }\end{array}$ & 15 & 17 & 20 & 5 & 16 & 18 \\
\hline LGI Dose & $\begin{array}{c}200 \mathrm{cGy} / \mathrm{d} \times 4 \\
\text { using } 6 \mathrm{MV} / 18 \mathrm{MV} \\
\text { photons }\end{array}$ & $\begin{array}{c}150 \mathrm{cGy} / \mathrm{d} \times 4 \\
\text { using } 18 \mathrm{MV} \\
\text { photons }\end{array}$ & $\begin{array}{l}150 \mathrm{cGy} / \mathrm{d} \times 4 \text { us- } \\
\text { ing } 6 \mathrm{MV} \text { photons }\end{array}$ & $\begin{array}{c}200 \mathrm{cGy} / \mathrm{d} \times 4 \\
\text { using } 6 \mathrm{MV} / 18 \mathrm{MV} \\
\text { photons }\end{array}$ & $\begin{array}{c}200 \mathrm{cGy} / \mathrm{d} \times 4 \\
\text { using } 6 \mathrm{MV} / 18 \mathrm{MV} \\
\text { photons }\end{array}$ & $\begin{array}{c}200 \mathrm{cGy} / \mathrm{d} \times 4 \\
\text { doses using } 6 \mathrm{MV} \\
\text { photons }\end{array}$ \\
\hline $\begin{array}{l}\text { Change in Se- } \\
\text { rum creatinine, } \\
\text { mg/dL within a } \\
\text { month post-LGI }\end{array}$ & $\begin{array}{l}\text { Serum Cr de- } \\
\text { creased from } 5.2 \\
\text { mg/dL to } 4.4 \mathrm{mg} / \\
\text { dL a month after } \\
\text { radiotherapy }\end{array}$ & $\begin{array}{l}\text { Serum Cr de- } \\
\text { creased from } 4.3 \\
\text { mg/dL to } 3.3 \mathrm{mg} / \\
\text { dL at } 2 \text { weeks }\end{array}$ & $\begin{array}{l}\text { Serum Cr de- } \\
\text { creased from } 5.9 \\
\text { mg/dL to } 4.8 \mathrm{mg} / \\
\text { dL at } 2 \text { weeks }\end{array}$ & $\begin{array}{l}\text { Serum Cr de- } \\
\text { creased from } 6.9 \\
\text { mg/dL to } 3.8 \mathrm{mg} / \\
\text { dL at } 3 \text { weeks }\end{array}$ & $\begin{array}{c}\text { Serum Cr re- } \\
\text { mained stable } \\
\text { between 3.9-4.1 } \\
\text { mg/dL. }\end{array}$ & $\begin{array}{c}\text { Serum Cr in- } \\
\text { creased from } 7.0 \\
\text { mg/dL to } 7.7 \mathrm{mg} / \\
\text { dL a week after } \\
\text { LGI }\end{array}$ \\
\hline $\begin{array}{l}\text { Outcome post } \\
\text { LGI }\end{array}$ & $\begin{array}{l}\text { Renal function } \\
\text { has remained } \\
\text { stable since LGI }\end{array}$ & $\begin{array}{l}\text { Renal function } \\
\text { stabilized for } \\
2 \text { months but } \\
\text { deteriorated at } 3 \\
\text { months post-LGI } \\
\text { and patient initi- } \\
\text { ated dialysis }\end{array}$ & $\begin{array}{l}\text { Renal function } \\
\text { stabilized for } \\
6 \text { months but } \\
\text { deteriorated at } 7 \\
\text { months post LGI } \\
\text { and patient initi- } \\
\text { ated dialysis }\end{array}$ & $\begin{array}{l}\text { Renal function } \\
\text { stabilized for } \\
2 \text { months but } \\
\text { deteriorated at } 3 \\
\text { months post LGI } \\
\text { and patient initi- } \\
\text { ated dialysis }\end{array}$ & $\begin{array}{c}\text { Renal function } \\
\text { stabilized for } 3 \\
\text { months and but } \\
\text { deteriorated at } 4 \\
\text { months post- LGI, } \\
\text { patient initiated } \\
\text { dialysis }\end{array}$ & $\begin{array}{l}\text { Renal function } \\
\text { did not improve } \\
\text { post-LGI therapy } \\
\text { and patient } \\
\text { initiated dialysis } 1 \\
\text { week post-LGI }\end{array}$ \\
\hline $\begin{array}{l}\text { Graft sur- } \\
\text { vival, post-LGI, } \\
\text { months }\end{array}$ & $\begin{array}{l}\text { Kidney allograft } \\
\text { functional till last } \\
\text { follow-up of } 24 \\
\text { months }\end{array}$ & 3 & 7 & 3 & 4 & 0 \\
\hline
\end{tabular}




\begin{tabular}{|c|c|c|c|c|c|c|}
\hline Author & $\begin{array}{c}\text { Number of } \\
\text { Patients }\end{array}$ & Selection Criteria & $\begin{array}{c}\text { Dose Range, } \\
\text { cGy }\end{array}$ & $\begin{array}{l}\text { Median Dose, } \\
\text { cGy }\end{array}$ & $\begin{array}{c}\text { One Month } \\
\text { Graft Survival } \\
\text { Post-LGI, } \%\end{array}$ & $\begin{array}{l}\text { One Year Graft } \\
\text { Survival Post- } \\
\text { LGI, } \%\end{array}$ \\
\hline \multirow[t]{2}{*}{ Halperin et al. (12) } & 53 & $\begin{array}{l}\text { Refractory acute allograft rejec- } \\
\text { tion }\end{array}$ & $300-1200$ & 600 & 42 & 21 \\
\hline & 10 & $\begin{array}{l}\text { Acute allograft rejection with con- } \\
\text { traindicated immunosuppressive } \\
\text { therapy due to systemic infection } \\
\text { or hematologic dyscrasias }\end{array}$ & $300-1200$ & 600 & 90 & 40 \\
\hline Jagetia et al. (13) & 6 & $\begin{array}{l}\text { Refractory acute allograft rejec- } \\
\text { tion }\end{array}$ & $450-600$ & 450 & 50 & 17 \\
\hline Noyes et al. (14) & 72 & $\begin{array}{c}\text { Refractory acute allograft rejec- } \\
\text { tion }\end{array}$ & 800 & 800 & NA & 49 \\
\hline Chen et al. (17) & 53 & $\begin{array}{l}\text { Refractory acute allograft rejec- } \\
\text { tion ( } 75 \% \text { of patients) }\end{array}$ & 600 & 600 & 83 & 60 \\
\hline Nuyttens et al. (15) & 20 & $\begin{array}{c}\text { Refractory acute allograft rejec- } \\
\text { tion }\end{array}$ & 450 & 450 & NA & 50 \\
\hline Wahl et al. (16) & 33 & $\begin{array}{l}\text { Refractory acute allograft rejec- } \\
\text { tion }\end{array}$ & 800 & 800 & 63 & 31 \\
\hline Current study & 6 & $\begin{array}{c}\text { Refractory acute allograft rejec- } \\
\text { tion }\end{array}$ & $800-600$ & 733 & 83 & 17 \\
\hline
\end{tabular}

a Abbreviations: LGI, local graft irradiation; NA, not available.

b Biopsy proven acute allograft kidney rejection resistant to medical therapy.

\section{Acknowledgements}

There is no acknowledgment.

\section{Author's Contribution}

All authors contributed to data collection and writing of the article.

\section{Financial Disclosure}

There is no financial disclosure.

\section{Funding/Support}

This study has no external source of funding or support.

\section{References}

1. Pascual M, Theruvath T, Kawai T, Tolkoff-Rubin N, Cosimi AB Strategies to improve long-term outcomes after renal transplantation. NEngl J Med. 2002;346(8):580-90.

2. Scherer MN, Banas B, Mantouvalou K, Schnitzbauer A, Obed A, Kramer BK, et al. Current concepts and perspectives of immunosuppression in organ transplantation. Langenbecks Arch Surg. 2007;392(5):511-23.

3. Heeger PS, Dinavahi R. Transplant immunology for non-immunologist. Mt Sinai J Med. 2012;79(3):376-87.

4. Corash L, Lin L. Novel processes for inactivation of leukocytes to prevent transfusion-associated graft-versus-host disease. Bone Marrow Transplant. 2004;33(1):1-7.

5. Hume DM, Magee JH, Kauffman HM, Jr., Rittenbury MS, Prout GR, Jr.. Renal Homotransplantation in Man in Modified Recipients. Ann Surg. 1963;158:608-44.
6. Birkeland SA, Moesner J, Wiik A. The immunosuppressive effect of extracorporeal irradiation of the blood evaluated by blast transformation and membrane immune fluorescence tests. Scand J Immunol. 1976;5(1-2):117-27.

7. Najarian JS, Ferguson RM, Sutherland DE, Slavin S, Kim T, Kersey $\mathrm{J}$, et al. Fractionated total lymphoid irradiation as preparative immunosuppression in high risk renal transplantation: clinical and immunological studies. Ann Surg. 1982;196(4):442-52.

8. Abramson N, Ornitz R, Cavanaugh PJ. The effect of local irradiation on rejection of the transplanted kidney. Radiology. 1974;112(2):413-6.

9. Godfrey AM, Salaman JR. Radiotherapy in treatment of acute rejection of human renal allografts. Lancet.1976;1(7966):938-9.

10. Pilepich MV, Sicard GA, Breaux SR, Etheredge EE, Blum J, Anderson CB. Renal graft irradiation in acute rejection. Transplantation. 1983;35(3):208-11

11. Torrisi JR, Dritschilo A, Harter KW, Helfrich B, Berg CD, Whitfield $G$, et al. A randomized study of the efficacy of adjuvant local graft irradiation following renal transplantation. Int J Radiat Oncol Biol Phys. 1990;18(5):1027-31.

12. Halperin EC, Delmonico FL, Nelson PW, Shipley WU, Cosimi AB The use of local allograft irradiation following renal transplantation. Int J Radiat Oncol Biol Phys. 1984;10(7):987-90.

13. Jagetia R, Small W, Jr., Stuart FP, Shetty R, Stuart J, Kanwar YS, et al. Local graft irradiation after failure of modern immunosuppression in acute cellular and vascular graft rejection. Int J Radiat Oncol Biol Phys. 1996;36(4):907-11.

14. Noyes WR, Rodriguez R, Knechtle SJ, Pirsch JD, Sollinger HW D'Alessandro AM, et al. Radiation therapy for renal transplant rejection refractory to pulse steroids and OKT3. Int J Radiat Oncol Biol Phys. 1996;34(5):1055-9.

15. Nuyttens JJ, Harper J, Jenrette JM, Turrisi AT, 3rd. Outcome of radiation therapy for renal transplant rejection refractory to chemical immunosuppression. Radiother Oncol. 2005;74(1):17-9.

16. Wahl AO, Small W, Jr., Dixler I, Strom S, Rademaker A, Leventhal J, et al. Radiotherapy for rejection of renal transplant allografts 


\section{Fallahzadeh MKet al.}

refractory to medical immunosuppression. Am J Clin Oncol. 2006;29(6):551-4.

17. Chen LM, Godinez J, Thisted RA, Woodle ES, Thistlewaite JR, Powers C, et al. New scoring system identifies kidney outcome with radiation therapy in acute renal allograft rejection. Int J Radiat
Oncol Biol Phys. 2000;46(4):999-1003.

18. Kumar V, Abbas AK, Fausto N, Aster JC. Robbins and Cotran pathologic basis of disease. Elsevier Health Sciences; 2009.

19. Abbas AK, Lichtman AH, Pillai S. Cellular and Molecular Immunology. Elsevier Health Sciences; 2011 